\title{
Fatores associados à sonolência excessiva diurna em pacientes de uma clínica do sono
}

\author{
Factors associated with excessive daytime sleepiness in sleep clinic patients
}

Factores asociados con la somnolencia diurna excesiva en pacientes de clínicas de sueño

Aline Rabelo Ferreira ${ }^{1}$, Sayonara da Silva Paniago ${ }^{1}$, Giuliana Macedo Mendes ${ }^{1}$, Antonio Márcio Teodoro Cordeiro Silva ${ }^{1}$, Rogério José de Almeida ${ }^{1 *}$.

\section{RESUMO}

Objetivo: Investigar os fatores associados com a Sonolência Excessiva Diurna (SED) em pacientes atendidos em uma clínica do sono. Métodos: Estudo transversal analítico realizado em uma clínica especializada em Medicina do Sono da cidade de Goiânia/GO. Pesquisa realizada por meio da aplicação de dois questionários, epidemiológico e Escala de Sonolência de Epworth (ESE), aos pacientes atendidos em uma clínica particular de Medicina do Sono, com faixa etária dos 18 a 60 anos e presença de algum Distúrbio do Sono. Foi adotado um nível de significância de $5 \%(P<0,05)$ nas análises estatísticas realizadas. Resultados: Foram entrevistados 50 pacientes, com idade média de 45,1 anos. A análise da escala ESE obteve uma média de 8,9 pontos, sendo que $38 \%$ apresentaram um escore acima de 10 pontos, o que indica SED. Apresentaram pontuação no ESE significativamente superior os pacientes tabagistas ( $P=0,03)$, os que faziam uso da leitura para pegar no sono $(P=0,01)$ e os que possuíam alguma doença respiratória $(P=0,02)$. Conclusão: Este estudo mostrou uma importante frequência de SED na amostra estudada, identificando-se diversos fatores associados à SED em pacientes da clínica investigada.

Palavras-Chave: Distúrbios do sono por sonolência excessiva, Medicina do sono, Sono.

\begin{abstract}
Objective: To investigate factors associated with daytime sleepiness (SED) in patients attending a sleep clinic. Methods: An analytical cross-sectional study performed at a clinic specialized in Sleep Medicine in the city of Goiânia/GO. This study was carried out through the application of two epidemiological questionnaires and Epworth (ESE) Sleepiness Scale to patients attending a private Sleep Medicine clinic, with ages ranging from 18 to 60 years and the presence of some Sleep Disorder. A significance level of $5 \%(P<0.05)$ was adopted in the statistical analyzes performed. Results: Fifty patients were interviewed, with a mean age of 45.1 years. The analysis of the ESE scale averaged 8.9 points, $38 \%$ of which presented a score above 10 points, indicating SED. There were significantly higher scores among the smokers $(P=0.03)$, those who used sleep reading $(P$ $=0.01)$ and those who had respiratory disease $(P=0.02)$. Conclusion: This study showed an important frequency of SED in the studied sample, identifying several factors associated with SED in patients of the investigated clinic.
\end{abstract}

Keywords: Sleep disorders due to excessive drowsiness, Sleep medicine, Sleep.

${ }^{1}$ Pontifícia Universidade Católica de Goiás (PUC Goiás). * E-mail: rogeriopucgo@gmail.com

SUBMETIDO EM: 11/2019

ACEITO EM: 12/2019

PUBLICADO EM: 2/2020 


\section{RESUMEN}

Objetivo: Investigar los factores asociados con la somnolencia diurna (SED) en pacientes que acuden a una clínica del sueño. Métodos: estudio transversal analítico realizado en una clínica especializada en medicina del sueño en la ciudad de Goiânia/GO. Investigación realizada mediante la aplicación de dos cuestionarios, Epidemiológico y Epworth Sleepiness Scale (ESE), a pacientes atendidos en una clínica privada de medicina del sueño, de 18 a 60 años y la presencia de algún trastorno del sueño. Se adoptó un nivel de significancia del $5 \%(P<0.05)$ en los análisis estadísticos realizados. Resultados: Entrevistamos a 50 pacientes, con una edad média de 45,1 años. El análisis de la escala ESE obtuvo un promedio de 8.9 puntos, y el $38 \%$ tuvo una puntuación superior a 10 puntos, lo que indica SED. Se encontraron puntuaciones de ESS significativamente más altas para los fumadores $(P=0.03)$, aquellos que usaron la lectura del sueño $(P=0.01)$ y aquellos con enfermedad respiratoria $(P=0.02)$. Conclusión: Este estudio mostró una frecuencia importante de SED en la muestra estudiada, identificando varios factores asociados con SED en pacientes de la clínica investigada.

Palabras clave: Trastornos del sueño debido a somnolencia excessiva, Medicina del sueño, Dormir.

\section{INTRODUÇÃO}

O sono é um fenômeno vital, tão necessário à manutenção da existência quanto o ato da alimentação. Dormir tem uma função biológica fundamental na consolidação da memória, normalização das funções endócrinas, termorregulação, conservação, restauração da energia, e na restauração do metabolismo energético cerebral (RIBEIRO CRF, et al., 2014).

O ciclo sono-vigília acompanha o ritmo circadiano, isto é, apresenta sincronização com fatores intrínsecos pessoais e ambientais no período de 24 horas. A alternância do dia-noite (claro-escuro), os horários escolares, de trabalho e de lazer, as atividades familiares, todos são fatores que sincronizam o ciclo sono-vigília. Esse ciclo é gerado e regulado endogenamente por uma estrutura neural localizada no hipotálamo, que é o núcleo supraquiasmático, considerado o relógio biológico para os mamíferos (RIBEIRO CRF, et al., 2014).

Dois grandes estágios compõem o sono do adulto, o NREM (75\%) e o REM (25\%), sendo que o estágio NREM é subdividido em três fases específicas. Na primeira parte do momento em que se fecha os olhos e 0 corpo começa a pegar no sono. Na segunda o corpo já se encontra relaxado e dormindo com a mente atenta. $\mathrm{Na}$ terceira tem-se o sono profundo em que os músculos relaxam completamente com a mente desligada. Já no sono REM os olhos se movimentam muito rapidamente, o batimento cardíaco aumenta e tem 0 aparecimento dos sonhos (NEVES GSML, et al., 2013).

Os Distúrbios do Sono compõem todas as alterações que ocorrem enquanto o indivíduo está dormindo. Pode-se identificar que as causas mais comuns de prejuízo do sono são a restrição e sua fragmentação (ARAÚJO JP e MELO HCS, 2016). A fragmentação resulta em um sono de quantidade e qualidade inadequadas, sendo consequência de condições biológicas e/ou fatores ambientais que o interrompem. Para um estado ótimo de vigília, o adulto requer uma média de 7 a 8 horas de sono em um período de 24 horas (RIBEIRO CRF, et al., 2014).

A última Classificação Internacional de Distúrbios do Sono (ICSD-3) identificou sete categorias principais: distúrbios de insônia, distúrbios respiratórios relacionados ao sono, distúrbios centrais da hipersonolência, distúrbios do sono-vigília do ritmo circadiano, distúrbios do movimento relacionados ao sono, parasonias, além de outros Distúrbios do Sono (INTERNATIONAL CLASSIFICATION OF SLEEP DISORDERS, 2014; SATEIA MJ, 2014).

Os Distúrbios do Sono tendem a provocar condições adversas na vida das pessoas contribuindo para a diminuição de seu funcionamento diário agravando problemas de saúde já existentes (MULLER MR, GUIMARÃES SS, 2007). A Síndrome da Apneia/Hipopneia Obstrutiva do Sono (SAHOS), por exemplo, que é um tipo comum de Distúrbio do Sono, é frequentemente associada a acidentes automobilísticos, domésticos e de trabalho, bem como ao agravamento ou início de doenças cardíacas que levam a um alto nível de 
morbimortalidade da população (PAMPOLIM G, et al., 2016). Foi relatado também uma forte associação entre o aumento da sonolência diurna nos indivíduos e os principais componentes da síndrome metabólica, como aumento da circunferência abdominal e hipertensão (MANSUR AP, et al., 2015).

A Sonolência Excessiva Diurna (SED) é uma das mais frequentes queixas relacionadas aos Distúrbios do Sono e pode ser definida como a incapacidade de se manter acordado e alerta durante o dia, resultando em sonolência e lapsos de sono não intencionais (NEVES GSML, et al., 2013). Os Distúrbios do Sono estão intrinsicamente relacionados com a SED, sendo que os principais fatores que contribuem são: duração do sono, qualidade do sono, horário de despertar, condições médicas e neurológicas associadas com impacto direto no sono (depressão, ansiedade, epilepsia, hipotireoidismo e anemia), medicamentos psicoativos e a presença de um transtorno primário do sono cursando com hipersonia (GIORELLI AS, et al., 2012). A SED foi considerada como sintoma subjetivo, muitas vezes não sendo reconhecido ou relegado a outras explicações pelos pacientes (BURGOS RA, CARVALHO GA, 2012).

O impacto da SED no trabalhador adulto pode se manifestar como diminuição de produtividade, aumento de absenteísmo, maiores índices de acidentes e uma maior probabilidade de incapacitação ocasionada pela doença (BERTOLAZI AN, et al., 2009).

Em um estudo, a SED foi encontrada em metade dos pacientes com SAHOS, apresentando baixos valores de sensibilidade e especificidade, mas também estava presente na maioria dos pacientes sem SAHOS (PAMPOLIM G, et al., 2016).

Portanto, o presente estudo teve por objetivo investigar os fatores associados com a sonolência excessiva diurna em pacientes atendidos em uma clínica do sono.

\section{MÉTODOS}

Trata-se de um Estudo transversal analítico com abordagem quantitativa. O estudo realizado em uma Clínica especializada em sono situada na cidade de Goiânia (GO), cuja coleta de dados ocorreu entre os meses de abril e junho de 2018.

Como critérios de inclusão, foram pesquisados os indivíduos entre 18 e 60 anos, de ambos os sexos, pacientes com algum transtorno do sono e que estavam na clínica no momento da pesquisa. Não foram incluídos os pacientes que referiram algum déficit cognitivo e que não responderam todas as questões dos questionários.

Para a realização do cálculo amostral foi utilizado o número de pacientes atendidos nos últimos dois meses (fevereiro e março de 2018) que antecederam a coleta de dados. Nesse período foram atendidos, dentro dos critérios de inclusão e exclusão, um total de 57 pacientes. Com esta população utilizou-se um nível de confiança de $95 \%$ e margem de erro de $5 \%$, com a amostra final composta por 50 pacientes, sendo utilizada uma amostragem por conveniência.

Foram utilizados dois instrumentos de pesquisa, sendo um questionário com amplitude epidemiológica, que procurou identificar variáveis socioculturais, pessoais e clínicas dos participantes, e o outro, para avaliação da SED, foi a Escala de Sonolência de Epworth (ESE), que é um instrumento desenvolvido em 1991 por um médico australiano, Dr. John W. Murray, e é utilizada desde então em todo o mundo, sendo já traduzida para vários idiomas, como o alemão, espanhol e japonês (BOARI L, et al., 2004).

Foi adaptado e validado no Brasil e tem por objetivo quantificar a propensão para adormecer durante oito situações rotineiras do dia-a-dia dos indivíduos. As respostas atingem valor máximo de 24 e mínimo de 0 pontos, sendo considerado o escore maior que 10 como diagnóstico de SED (BERTOLAZI AN, et al., 2009).

Sua mensuração não é subjetiva, apesar de depender da habilidade de leitura, compreensão e resposta honesta do paciente. A ESE tem servido, ao longo dos anos, de triagem para os Distúrbios do Sono e como um importante indicador para a execução do exame de polissonografia (BOARI L, et al., 2004). 
As análises estatísticas foram realizadas com o auxílio pacote estatístico SPSS 24 (Statistical Package of Social Sciences). A caracterização do perfil sociodemográfico, pessoal e clínico foi realizada por meio de frequência absoluta $(n)$ e relativa $(\%)$.

A normalidade dos dados foi verificada utilizando o teste de D'Agostino-Pearson. Para as variáveis que foram constatadas a normalidade foram utilizados os testes de $t$ e de ANOVA. Já para as que não apresentaram normalidade utilizaram-se os testes de Mann-Whitney e Kruskal-Wallis. Em todas as análises foi adotado um nível de significância de $5 \%(p<0,05)$.

O estudo foi aprovado pelo Comitê de Ética em Pesquisa (CEP) da Pontifícia Universidade Católica de Goiás (PUC Goiás), sob o parecer no 2.404.422, CAAE: 79846117.0.0000.0037. Todos os participantes leram e concordaram com a pesquisa assinando o Termo de Consentimento Livre e Esclarecido.

\section{RESULTADOS}

Foram entrevistados 50 pacientes com algum Distúrbio do Sono, atendidos em uma clínica especializada na cidade de Goiânia/GO. A análise da SED por meio da ESE mostrou que $38 \%$ dos pacientes apresentaram um escore acima de 10 pontos, o que indica SED. O escore médio obtido no conjunto da amostra investigada foi de 8,9 pontos (Tabela $\mathbf{1}$ ).

Tabela 1 - Análise da Sonolência Excessiva Diurna (SED) medida pela Escala de Sonolência de Epworth dos 50 indivíduos com algum Distúrbio do Sono, Goiânia, Goiás, Brasil, 2018.

\begin{tabular}{lcc}
\hline Variáveis & $\boldsymbol{N}$ & $\%$ \\
\hline Sonolência Excessiva Diurna & & \\
$\leq 10$ & 31 & 62,0 \\
$>10$ & 19 & 38,0 \\
Escore Médio e DP & 8,9 & 4,7 \\
\hline
\end{tabular}

\footnotetext{
Legenda:

$\mathrm{N}$ = Frequência absoluta;

$\%=$ Frequência
}

Fonte: Ferreira AR, et al., 2019.

Em relação aos aspectos socioculturais dos pacientes estudados, a idade teve uma média de 45,1 anos, sendo que $50 \%$ eram homens e $50 \%$ mulheres, $34 \%$ eram casados/união estável, $74 \%$ possuíam ensino superior completo/incompleto, $72 \%$ detinham uma renda pessoal acima de quatro salários mínimos, $48 \%$ dos pacientes faziam ingestão de bebida alcóolica e $94 \%$ negaram tabagismo. A análise comparativa dos aspectos socioculturais com a SED demonstrou-se que os pacientes tabagistas apresentaram pontuação no ESE superior $(13,7 \pm 3,2)$ aos não tabagistas $(8,6 \pm 4,6)(P=0,03)$ (Tabela 2). 
Tabela 2 - Comparação da Sonolência Excessiva Diurna com os aspectos socioculturais dos 50 indivíduos com algum Distúrbio do Sono, Goiânia, Goiás, Brasil, 2018.

\begin{tabular}{|c|c|c|c|c|c|}
\hline Variáveis & $N$ & $\%$ & $\begin{array}{l}\text { Média } \\
\text { (ESE) }\end{array}$ & DP & $p$-valor \\
\hline \multicolumn{6}{|l|}{ Sexo } \\
\hline Masculino & 25 & 50,0 & 9,7 & 4,7 & \\
\hline Feminino & 25 & 50,0 & 8,1 & 4,6 & 0,122 \\
\hline \multicolumn{6}{|l|}{ Idade (anos) } \\
\hline$\leq 50$ & 29 & 58,0 & 9,4 & 4,5 & \\
\hline$>50$ & 21 & 42,0 & 8,2 & 5,0 & 0,185 \\
\hline Média (DP) & 45,1 & 12,4 & & & \\
\hline \multicolumn{6}{|l|}{ Estado Civil } \\
\hline Solteiro & 27 & 54,0 & 9,4 & 4,7 & \\
\hline Casado/União Estável & 17 & 34,0 & 9,4 & 4,3 & \\
\hline Divorciado & 2 & 4,0 & 1,0 & 1,4 & \\
\hline Viúvo & 4 & 8,0 & 7,5 & 4,8 & 0,085 \\
\hline \multicolumn{6}{|l|}{ Cor Autorreferida } \\
\hline Branca & 30 & 60,0 & 9,5 & 4,4 & \\
\hline Parda & 18 & 36,0 & 7,9 & 4,8 & \\
\hline Amarela & 2 & 4,0 & 9,0 & 9,9 & 0,569 \\
\hline \multicolumn{6}{|l|}{ Escolaridade } \\
\hline Ensino Fundamental & 3 & 6,0 & 8,0 & 4,6 & \\
\hline Ensino Médio & 10 & 20,0 & 7,1 & 3,1 & \\
\hline Ensino Superior completo/incompleto & 37 & 74,0 & 9,5 & 5,0 & 0,358 \\
\hline \multicolumn{6}{|l|}{ Religião } \\
\hline $\operatorname{Sim}$ & 44 & 88,0 & 8,7 & 4,7 & \\
\hline Não & 6 & 12,0 & 10,7 & 4,9 & 0,166 \\
\hline \multicolumn{6}{|l|}{ Renda Pessoal } \\
\hline Abaixo de um salário mínimo & 4 & 8,0 & 5,0 & 1,6 & \\
\hline De 1 a 4 salários mínimos & 10 & 20,0 & 9,7 & 4,8 & \\
\hline Acima de 4 salários mínimos & 36 & 72,0 & 9,1 & 4,8 & 0,212 \\
\hline \multicolumn{6}{|l|}{ Atividade de Lazer } \\
\hline $\operatorname{Sim}$ & 29 & 58,0 & 9,8 & 5,0 & \\
\hline Não & 21 & 42,0 & 7,7 & 4,1 & 0,065 \\
\hline \multicolumn{6}{|l|}{ Utilizar a Internet } \\
\hline Sim & 46 & 92,0 & 9,1 & 4,6 & \\
\hline Não & 4 & 8,0 & 6,3 & 5,4 & 0,122 \\
\hline \multicolumn{6}{|l|}{ Ingestão de Álcool } \\
\hline $\operatorname{Sim}$ & 24 & 48,0 & 8,7 & 4,6 & \\
\hline Não & 26 & 52,0 & 9,1 & 4,8 & 0,062 \\
\hline \multicolumn{6}{|l|}{ É fumante } \\
\hline Sim & 3 & 6,0 & 13,7 & 3,2 & \\
\hline Não & 47 & 94,0 & 8,6 & 4,6 & $0.035^{\star}$ \\
\hline
\end{tabular}

Legenda: $\mathrm{N}$ = Frequência absoluta; \% = Frequência relativa; $\mathrm{DP}$ = Desvio padrão; Média (ESE) = Escore médio da escala de Epworth. Fonte: Ferreira AR, et al., 2019. 
Sobre os aspectos pessoais, $66 \%$ dos pacientes praticavam atividade física, $72 \%$ faziam uso de algum medicamento, $88 \%$ se consideravam estressados/ansiosos, $74 \%$ roncavam durante o sono e $10 \%$ utilizavam a leitura para pegar no sono. Comparando os aspectos pessoais com a SED identificou-se que os pacientes que faziam uso da leitura para pegar no sono tiveram pontuação no ESE superior $(11,6 \pm 5,8)$ aqueles que se utilizavam de TV $(11,1 \pm 4,2)$, música $(5,8 \pm 3,2)$, medicamentos $(4,7 \pm 4,0)$ e outros recursos $(7,6 \pm 4,2)(P=0,01)$ (Tabela 3).

Tabela 3 - Comparação da Sonolência Excessiva Diurna com os aspectos pessoais dos 50 indivíduos com algum Distúrbio do Sono, Goiânia, Goiás, Brasil, 2018.

\begin{tabular}{|c|c|c|c|c|c|}
\hline Variáveis & $N$ & $\%$ & $\begin{array}{l}\text { Média } \\
\text { (ESE) }\end{array}$ & DP & $p$-valor \\
\hline \multicolumn{6}{|c|}{ Pratica Atividade Física } \\
\hline $\operatorname{Sim}$ & 33 & 66,0 & 9,2 & 4,8 & \\
\hline Não & 17 & 34,0 & 8,4 & 4,7 & 0,280 \\
\hline \multicolumn{6}{|c|}{ Usa Algum Medicamento } \\
\hline $\operatorname{Sim}$ & 36 & 72,0 & 9,3 & 5,0 & \\
\hline Não & 14 & 28,0 & 8,0 & 4,0 & 0,202 \\
\hline \multicolumn{6}{|c|}{ Se Considerada Estressada/Ansiosa } \\
\hline $\operatorname{Sim}$ & 44 & 88,0 & 8,9 & 4,6 & \\
\hline Não & 6 & 12,0 & 8,7 & 6,0 & 0,449 \\
\hline \multicolumn{6}{|l|}{ Se Considerada Triste } \\
\hline Sim & 8 & 16,0 & 7,6 & 5,0 & \\
\hline Não & 42 & 84,0 & 9,1 & 4,7 & 0,204 \\
\hline \multicolumn{6}{|l|}{ Ronca Durante o Sono } \\
\hline $\operatorname{Sim}$ & 37 & 74,0 & 8,9 & 4,8 & \\
\hline Não & 9 & 18,0 & 9,9 & 5,1 & \\
\hline Não sei & 4 & 8,0 & 6,5 & 3,3 & 0,295 \\
\hline \multicolumn{6}{|l|}{ Tem Sono Muito Leve } \\
\hline Sim & 23 & 46,0 & 9,3 & 4,3 & \\
\hline Não & 27 & 54,0 & 8,6 & 5,1 & 0,311 \\
\hline \multicolumn{6}{|c|}{ Atividade para Pegar no Sono } \\
\hline TV & 18 & 36,0 & 11,1 & 4,2 & \\
\hline Leitura & 5 & 10,0 & 11,6 & 5,8 & \\
\hline Música & 5 & 10,0 & 5,8 & 3,2 & \\
\hline Medicamento & 3 & 6,0 & 4,7 & 4,0 & \\
\hline Outros & 19 & 38,0 & 7,6 & 4,2 & $0.013^{\star}$ \\
\hline \multicolumn{6}{|c|}{ Tempo que leva para dormir } \\
\hline Até 30 minutos & 31 & 62,0 & 9,7 & 4,8 & \\
\hline 31 a 60 minutos & 17 & 34,0 & 7,1 & 3,8 & \\
\hline Mais de 60 minutos & 2 & 4,0 & 11,0 & 8,5 & 0,145 \\
\hline \multicolumn{6}{|c|}{ Fácil voltar a dormir após acordar } \\
\hline $\operatorname{Sim}$ & 28 & 56,0 & 8,8 & 4,1 & \\
\hline Não & 22 & 44,0 & 9,0 & 5,5 & 0,424 \\
\hline
\end{tabular}

Legenda: $\mathrm{N}$ = Frequência absoluta; \% = Frequência relativa; DP = Desvio padrão; Média (ESE) = Escore médio da escala de Epworth.

Fonte: Ferreira AR, et al., 2019. 
Quanto aos aspectos clínicos, 38\% dos pacientes tinham alguma doença crônica, 4\% doença respiratória, $16 \%$ doença neurológica, $62 \%$ acordavam com a boca seca, $76 \%$ apresentavam diminuição da memória e $70 \%$ relataram diminuição da capacidade de concentração. A comparação entre os aspectos clínicos e a SED demonstrou que pacientes com alguma doença respiratória tiveram uma pontuação no ESE superior $(15,5 \pm 3,5)$ aos que não possuíam doença respiratória $(8,6 \pm 4,6)(P=0,02)$ (Tabela 4).

Tabela 4 - Comparação da Sonolência Excessiva Diurna com os aspectos clínicos 50 indivíduos com algum Distúrbio do Sono, Goiânia, Goiás, Brasil, 2018.

\begin{tabular}{|c|c|c|c|c|c|}
\hline Variáveis & $N$ & $\%$ & $\begin{array}{l}\text { Média } \\
\text { (ESE) }\end{array}$ & DP & $p$-valor \\
\hline \multicolumn{6}{|c|}{ Possui alguma Doença Crônica } \\
\hline $\operatorname{Sim}$ & 19 & 38,0 & 8,9 & 5,2 & \\
\hline Não & 31 & 62,0 & 8,9 & 4,5 & 0,478 \\
\hline \multicolumn{6}{|c|}{ Possui alguma Doença Neurológica } \\
\hline Sim & 8 & 16,0 & 10,1 & 4,8 & \\
\hline Não & 42 & 84,0 & 8,7 & 4,7 & 0,213 \\
\hline \multicolumn{6}{|c|}{ Possui alguma Doença Respiratória } \\
\hline Sim & 2 & 4,0 & 15,5 & 3,5 & \\
\hline Não & 48 & 96,0 & 8,6 & 4,6 & $0.021^{*}$ \\
\hline \multicolumn{6}{|c|}{ Possui alguma Doença do Coração } \\
\hline Sim & 6 & 12,0 & 10,8 & 6,2 & \\
\hline Não & 44 & 88,0 & 8,6 & 4,5 & 0,144 \\
\hline \multicolumn{6}{|c|}{ Acorda com sensação de engasgo } \\
\hline Sim & 17 & 34,0 & 9,6 & 4,7 & \\
\hline Não & 33 & 66,0 & 8,5 & 4,7 & 0,213 \\
\hline \multicolumn{6}{|c|}{ Dificuldade de respirar pelo nariz } \\
\hline Sim & 19 & 38,0 & 9,6 & 5,3 & \\
\hline Não & 31 & 62,0 & 8,5 & 4,3 & 0,215 \\
\hline \multicolumn{6}{|c|}{ Acorda com a boca seca } \\
\hline Sim & 31 & 62,0 & 9,1 & 5,3 & \\
\hline Não & 19 & 38,0 & 8,6 & 3,5 & 0,378 \\
\hline \multicolumn{6}{|c|}{ Range, aperta ou bate os dentes } \\
\hline Sim & 18 & 36,0 & 9,1 & 4,6 & \\
\hline Não & 32 & 64,0 & 8,8 & 4,8 & 0,407 \\
\hline \multicolumn{6}{|c|}{ Apresenta diminuição de memória } \\
\hline Sim & 38 & 76,0 & 9,0 & 4,9 & \\
\hline Não & 12 & 24,0 & 8,6 & 4,2 & 0,396 \\
\hline \multicolumn{6}{|c|}{ Apresenta diminuição de concentração } \\
\hline Sim & 35 & 70,0 & 9,1 & 4,6 & \\
\hline Não & 15 & 30,0 & 8,5 & 5,0 & 0,337 \\
\hline \multicolumn{6}{|c|}{ Apresenta Dor de Cabeça ao Acordar } \\
\hline Sim & 21 & 42,0 & 8,1 & 4,9 & \\
\hline Não & 29 & 58,0 & 9,5 & 4,5 & 0,154 \\
\hline
\end{tabular}

Legenda: $\mathrm{N}$ = Frequência absoluta; \% = Frequência relativa; DP = Desvio padrão; Média (ESE) = Escore médio da escala de Epworth; * Estatística inferencial: Teste G.

Fonte: Ferreira AR, et al., 2019. 


\section{DISCUSSÃO}

A literatura científica já demonstrou uma intensa associação entre Sonolência Excessiva Diurna e Distúrbios do Sono. A SED é um problema generalizado que os clínicos encontram com muita frequência em sua prática, sendo altamente prevalente, afetando até $30 \%$ do público em geral, e está associada a sequelas pessoais e ocupacionais significativas (STEVENSON MR, et al., 2014). Uma das causas mais comuns de SED é o Distúrbio Respiratório do Sono, sendo a SAHOS o mais frequente (GUAITA M, et al., 2015). No presente estudo, identificou-se que um total de $38 \%$ dos indivíduos investigados com algum Distúrbio do Sono apresentou escore acima da normalidade na Escala de Sonolência de Epworth, o que indica SED.

A frequência encontrada no estudo reforça a ideia de que os Distúrbios do Sono se configuram como uma gama de condições clínicas e sociais capazes de interferir negativamente em todos os órgãos e sistemas. As consequências podem ser inúmeras e não limitadas à fase em que o indivíduo está dormindo. As alterações do estilo de vida que vêm ocorrendo ao longo dos anos têm contribuído sobremaneira para que os indivíduos durmam menos. Outro problema é o aumento de pessoas com excesso de peso, fazendo com que desenvolva cada vez mais distúrbios respiratórios do sono (DRAGER LF, et al., 2018).

Em relação ao tabagismo, aqueles que fumavam apresentaram uma média de sonolência patológica. Uma forte associação entre tabagismo e SAHOS tem sido demonstrada em estudos observacionais (KRISHNAN $\mathrm{V}$, et al., 2014). Além disso, há um efeito sinérgico entre o tabagismo e a SAOS causando aumento da morbidade cardiovascular (DELEANU OC, et al., 2016).

Alguns mecanismos são sugeridos para explicar como o tabagismo pode causar SAHOS: mudanças na arquitetura do sono, relaxamento dos músculos das vias aéreas superiores e reflexos neurais causados pela nicotina, aumento do limiar de excitação do sono causada pela nicotina, e aumento da inflamação das vias aéreas superiores devido à inalação de fumaça (KRISHNAN V, et al., 2014). Portanto, alguns Distúrbios do Sono podem resultar de interações complexas entre os efeitos estimulantes da nicotina e os efeitos respiratórios do tabagismo (COSTA M e ESTEVES M, 2018).

A exposição crônica ao tabagismo leva à diminuição da sensibilidade à hipóxia com o funcionamento anormal dos quimiorreceptores periféricos, o que facilita durações mais longas das apneias com dessaturações e flutuação de oxigênio, tendo um papel direto no desenvolvimento de SED em pacientes com SAHOS grave (PUIU L, et al., 2014).

Este estudo demonstrou maiores escores da ESE em indivíduos que usam a leitura como atividade para pegar no sono. Estudos anteriores mostraram que pessoas que leem livros em aparelhos eletrônicos, em comparação com aqueles que o fazem em livros impressos, levam mais tempo para pegar no sono, têm uma regulação mais tardia do ciclo circadiano e diminuição do estado de alerta no dia seguinte, além de terem alteração na secreção hormonal de melatonina (CHANG AM, et al., 2015), resultando em uma duração mais curta do sono e aumento da sonolência diurna (NASIRUDEEN AMA, et al., 2017).

A relação entre o uso demasiado de dispositivos tecnológicos, como televisão, computadores ou telefones celulares e a SED já é uma evidência. O uso constante de smartphones e tablets, por exemplo, pode causar insônia, aumento de peso e inclusive doenças sistêmicas. A exposição luminosa a telefones e computadores portáteis diminui a produção orgânica do hormônio regulador do sono: a melatonina, o que pode causar e perpetuar a insônia, especialmente em adolescentes e adultos jovens, pois se tratam de indivíduos com tendência a adormecer mais tarde, ou seja, apresentam atraso de fase de sono (PERLOW LA, 2012).

O presente estudo revelou que pacientes que faziam uso de medicação para pegar no sono tiveram um escore bem abaixo daqueles que utilizaram a leitura e televisão. Importante nesse contexto identificar que 0 uso de medicação indutora de sono atua diminuindo queixas de SED (NEVES GSML, et al., 2013).

Os medicamentos-padrão para controlar a insônia agem por via GABAérgica (zolpidem, zopiclona) ou melatoninérgica central (ramelteon). Mais especificamente, os médicos têm prescrito vários medicamentos off-label para a insônia, como antidepressivos tricíclicos, benzodiazepínicos e antipsicóticos atípicos (GULYANI S, et al., 2012). 
Os dados deste trabalho demonstraram maior relação de SED associado a Distúrbio do Sono nos indivíduos com alguma doença respiratória. Estudos anteriores trazem essa associação principalmente com relação à Rinosinusite Crônica (RSC), Asma e Doença Pulmonar Obstrutiva Crônica (DPOC). Na RSC é possível que a inflamação das narinas e seios da face, além da obstrução nasal, gere bloqueio das vias aéreas, contribindo muito para o aparecimento de Distúrbio Respiratório do Sono (FERRANDO M, et al., 2016). Pacientes com RSC comumente sofrem de sintomas de dor facial e pressão relacionada à congestão crônica e inflamação dos seios paranasais. A dor crônica tem sido associada à depressão e também contribui para comprometer a qualidade e continuidade do sono (COX DR, et al., 2016).

Há um alto risco de SAHOS em pacientes com asma (PRASAD B, et al., 2014). Pacientes asmáticos têm um risco significativamente maior de desenvolver SAHOS do que a população geral (SHEN TC, et al., 2015). Parece haver também uma relação entre insônia e asma. A insônia é altamente prevalente em adultos asmáticos e quando presente está associada a um pior controle da asma. Além disso, há uma maior prevalência de depressão e sintomas ansiosos, afetando a qualidade de vida destes pacientes asmáticos (LUYSTER FS, et al., 2016).

Ainda neste contexto, distúrbios respiratórios noturnos em pacientes com Doença Pulmonar Obstrutiva Crônica (DPOC) podem estar associados à SED (ENZ C, et al., 2016). A DPOC é uma das principais causas de morbidade e mortalidade e pode ser frequentemente complicada por Distúrbios do Sono. A insônia e a SAHOS são comumente encontradas em pacientes com DPOC (BUDHIRAJA R, et al., 2015). Além disso, é sabido que a SAHOS é considerada fator de risco independente para hipertensão arterial, insuficiência cardíaca, doença arterial coronária e arritmias (CAMPOSTRINI DAC, et al., 2014).

Como forma de aprofundar a investigação sobre as possíveis causas subjacentes da SED, o profissional de saúde pode solicitar que o paciente preencha um diário de sono e relate suas rotinas. A partir desses dados, poderá orientar mudanças de comportamentos e de hábitos inadequados relacionados ao sono, além de encaminhar o indivíduo para uma avaliação por médico especializado em Medicina do Sono e, caso necessário, ser submetido ao exame de polissonografia, que consiste no registro de várias variáveis simultâneas durante o sono noturno (ANDRECHUK CRS e CEOLIM MF, 2015).

\section{CONCLUSÃO}

Em conclusão, os resultados deste estudo revelaram uma importante frequência de SED na amostra analisada. Além disso, o estudo demonstrou uma significativa associação entre SED e tabagismo, nas atividades desenvolvidas pelos pacientes para pegar no sono e também com as doenças respiratórias. Esses resultados, além de pouco estudados pela literatura, são de particular importância, uma vez que permitem melhorar a abordagem diagnóstica e terapêutica desses pacientes.

\section{REFERÊNCIAS}

1. ANDRECHUK CRS, CEOLIM MF. Sonolência diurna excessiva nos pacientes com infarto agudo do miocárdio. Acta Paulista de Enfermagem, 2015; 28(3): 230-236.

2. ARAÚJO JP, MELO HCS. Distúrbios do sono e transtornos de aprendizagem. Revista Científica Multidisciplinar Núcleo do Conhecimento. 2016; 10(1):102-113.

3. BERTOLAZI AN, et al. Validação da escala de sonolência de Epworth em português para uso no brasil. Jornal Brasileiro de Pneumologia, 2009; 35(9): 877-883.

4. BOARI L, et al. Avaliação da escala de Epworth em pacientes com a síndrome da apneia e hipopneia obstrutiva do sono. Revista Brasileira de Otorrinolaringologia, 2004; 70(6): 752-756.

5. BUDHIRAJA R, et al. Sleep disorders in chronic obstructive pulmonary disease: etiology, impact, and management. Journal of Clinical Sleep Medicine, 2015;11(3): 259-270.

6. BURGOS RA, CARVALHO GA. Síndrome da apneia obstrutiva do sono (SAOS) e sonolência diurna excessiva (SED): influência sobre os riscos e eventos de queda em idosos. Fisioterapia e Movimento, 2012; 25: 93-103.

7. CAMPOSTRINI DAC, et al. Síndrome da apneia obstrutiva do sono e doenças cardiovasculares. Revista de Neurociência, 2014; 22(1): 102-112. 
8. CHANG AM, et al. Evening use of light-emitting eReaders negatively affects sleep, circadian timing, and next-morning alerteness. Proceedings of the National Academy of Sciences, 2015; 112:1232-7.

9. COSTA M, ESTEVES M. Cigarette smoking and sleep disturbance. Addictive Disorders \& Their Treatment, 2018; $17(1): 40-48$.

10. COX DR, et al. Dyad of pain and depression in chronic rhinosinusitis. International Forum Allergy \& Rhinology, 2016;6: 308-314.

11. DELEANU OC, et al. Influence of smoking on sleep and obstructive sleep apnea syndrome. Pneumologia (Bucharest, Romania), 2016; 65:28-35.

12. DRAGER LF, et al. 1ํ Posicionamento Brasileiro sobre o Impacto dos Distúrbios de Sono nas Doenças Cardiovasculares da Sociedade Brasileira de Cardiologia. Arquivos Brasileiros de Cardiologia, 2018; 111(2):290340.

13. ENZ C, et al. Predictors of increased daytime sleepiness in patients with chronic obstructive pulmonary disease: a cross-sectional study. Sleep Disorder, 2016; e1089196.

14. FERRANDO $\mathrm{M}$, et al. Sleep complaints and sleep breathing disorders in upper and lower obstructive lung diseases. Journal of Thoracic Disease, 2016;8(8): 716-725.

15. GIORELLI AS, et al. Sonolência excessiva diurna: aspectos clínicos, diagnósticos e terapêuticos. Revista Brasileira de Neurologia, 2012; 48(3): 17-24.

16. GUAITA M, et al. The Barcelona Sleepiness Index: a new instrument to assess excessive daytime sleepiness in sleep disordered breathing. Journal of Clinical Sleep Medicine, 2015;11(11):1289-98.

17. GULYANI S, SALAS RE, GAMALDO CE. Sleep medicine pharmacotherapeutics overview: today, tomorrow, and the future (Part 1: Insomnia and circadian rhythm disorders). Chest, 2012; 142(6): 1659-68.

18. INTERNATIONAL CLASSIFICATION OF SLEEP DISORDERS - ICSD. 3. ed. Darien: American Academy of Sleep Medicine, 2014.

19. KRISHNAN V, et al. Where there is smoke...there is sleep apnea: exploring the relationship between smoking and sleep apnea. Chest, 2014; 146(6):1673-1680.

20. LUYSTER FS, et al. Association between insomnia and asthma burden in the severe asthma research program (SARP) III. Chest, 2016;150(6): 1242-1250.

21. MANSUR AP, et al. Fatores de risco para doença cardiovascular, síndrome metabólica e sonolência em motoristas de caminhão. Arquivos Brasileiros de Cardiologia, 2015; 105(6):560-565.

22. MULLER MR, GUIMARÃES SS. Impacto dos transtornos do sono sobre o funcionamento diário e a qualidade de vida. Estudos de Psicologia, 2007; 24(4): 519-528.

23. NASIRUDEEN AMA, et al. Impact of social media usage on daytime sleepiness: a study in a sample of tertiary students in Singapore. Digital Health, 2017; 3: 1-9.

24. NEVES GSML, et al. Transtornos do sono: visão geral. Revista Brasileira de Neurologia, $2013 ; 49$ (2): 57-71.

25. PAMPOLIM G, et al. Sonolência excessiva diurna em indivíduos com distúrbios respiratórios do sono. Journal of Health Science, 2016; 2 (2):1-10.

26. PERLOW LA. Sleeping with your smartphone: how to break the $24 / 7$ habit and change the way you work. Boston: Harvard Business Press Books, 2012.

27. PRASAD B, et al. Obstructive sleep apnea and asthma: associations and treatment implications. Sleep Medicine Reviews, 2014; 18(2): 165-71.

28. PUIU L, et al. Association of excessive daytime sleepiness with smoking, inflammatory markers and cardiovascular risk. European Sleep Research Society Congress, 2014.

29. RIBEIRO CRF, et al. O impacto da qualidade do sono na formação médica. Revista da Sociedade Brasileira de Clínica Médica, 2014; 12(1): 8-14.

30. SATEIA MJ. International classification of sleep disorders-third edition- highlights and modifications. Chest, 2014; 146(5): 1387-1394.

31. SHEN TC, et al. Risk of obstructive sleep apnea in adult patients with asthma: a population-based cohort study in Taiwan. PLoS ONE, 2015;10(6).

32. STEVENSON MR, et al. The role of sleepiness, sleep disorders, and the work environment on heavy-vehicle crashes in 2 Australian States. American Journal of Epidemiology, 2014; 179(5): 594-601. 